\title{
Almost Sure Asymptotical Adaptive Synchronization for Neutral-Type Neural Networks with Stochastic Perturbation and Markovian Switching
}

\author{
Wuneng Zhou, Xueqing Yang, Jun Yang, Anding Dai, and Huashan Liu \\ College of Information Sciences and Technology, Donghua University, Shanghai 200051, China \\ Correspondence should be addressed to Wuneng Zhou; zhouwuneng@163.com
}

Received 16 December 2013; Accepted 21 February 2014; Published 16 April 2014

Academic Editor: Zhengguang Wu

Copyright (c) 2014 Wuneng Zhou et al. This is an open access article distributed under the Creative Commons Attribution License, which permits unrestricted use, distribution, and reproduction in any medium, provided the original work is properly cited.

The problem of almost sure (a.s.) asymptotic adaptive synchronization for neutral-type neural networks with stochastic perturbation and Markovian switching is researched. Firstly, we proposed a new criterion of a.s. asymptotic stability for a general neutral-type stochastic differential equation which extends the existing results. Secondly, based upon this stability criterion, by making use of Lyapunov functional method and designing an adaptive controller, we obtained a condition of a.s. asymptotic adaptive synchronization for neutral-type neural networks with stochastic perturbation and Markovian switching. The synchronization condition is expressed as linear matrix inequality which can be easily solved by Matlab. Finally, we introduced a numerical example to illustrate the effectiveness of the method and result obtained in this paper.

\section{Introduction}

As it is well known, the stability and synchronization of neural networks can be applied to create chemical and biological systems, secure communication systems, information science, image processing, and so on. In recent years, different control methods are derived to achieve different synchronization, such as randomly occurring control [1], sampled-data control $[2,3]$, passivity analysis [4], impulsive control [5-8], and adaptive control [9].

By utilizing adaptive control method, the parameters of the system need to be estimated and the control law needs to be updated when the neural networks evolve. In the past decade, much attention has been devoted to the research of the adaptive synchronization for neural networks. In $[9,10]$, the adaptive lag synchronization of unknown chaotic neural networks is considered. Adaptive synchronization problem of delayed neural networks with stochastic perturbation is studied in [11]. Besides these, there are many literatures to study adaptive synchronization problems (see, e.g., $[12,13]$ and the references therein).

Recently, the stability and synchronization of neutraltype systems, specially neutral-type neural networks, which depend on the derivative of the state and the delay state have attracted a lot of attention (see, e.g., [14-19] and the references therein) due to the fact that some physical systems in the real world can be described by neutral-type models (see [20]). However, the adaptive control was not investigated in [14-17], and the neutral term of derivative of the delay state was not taken into account in the neural networks proposed in [9-13]. Zhou et al. in [18] did not study the almost sure (a.s.) synchronization for neutral-type neural networks. Zhu et al. in [19] did not research the synchronization problem for neural networks with Markovian switching parameters. From the authors' best knowledge, so far the almost surely adaptive synchronization problem for neutral-type neural networks with stochastic perturbation and Markovian switching parameters has not been fully investigated yet. This motivates our current work.

In this paper, the problem of almost sure (a.s.) asymptotic adaptive synchronization for neutral-type neural networks with stochastic perturbation and Markovian switching is researched. By making use of Lyapunov functional method and designing an adaptive controller, we obtained a condition of a.s. asymptotic adaptive synchronization for neutral-type 
neural networks with stochastic perturbation and Markovian switching. Finally, we introduced a numerical example to illustrate the effectiveness of the method and result obtained in this paper. The main contributions of this paper are as follows.

(1) A new model for a class of neutral-type neural networks with stochastic perturbation and Markovian switching is given; it is more general than other models.

(2) A new criterion of a.s. asymptotic stability for a general neutral-type stochastic differential equation is proposed which extends the existing results.

The notations are quite standard. Throughout this paper, $\mathbb{R}_{+}, \mathbb{R}^{n}$, and $\mathbb{R}^{n \times n}$ denote the set of nonnegative real numbers, $n$-dimensional Euclidean space, and the set of all $n \times n$ real matrices, respectively. The superscript $T$ denotes matrix transposition, trace $(\cdot)$ denotes the trace of the corresponding matrix, and $I$ denotes the identity matrix. $|\cdot|$ stands for the Euclidean norm in $\mathbb{R}^{n}$. $\operatorname{diag}\{\cdots\}$ stands for the block diagonal matrix. Let $(\Omega, \mathscr{F}, \mathbb{P})$ be a complete probability space with a filtration $\left\{\mathscr{F}_{t}\right\}_{t \geq 0}$ satisfying the usual conditions (i.e., the filtration contains all $P$-null sets and is increasing and right continuous). For $p>0$, denote by $\mathbb{L}_{\mathscr{F}_{0}}^{p}\left([-\tau, 0] ; \mathbb{R}^{n}\right)$ the family of all $\mathscr{F}_{0}$-measurable, $\mathbb{C}\left([-\tau, 0] ; \mathbb{R}^{n}\right)$-valued random variables $\xi$ such that the mathematical expectation $\mathbb{E}\|\xi\|^{p}<$ $\infty$, where $\mathbb{C}\left([-\tau, 0] ; \mathbb{R}^{n}\right)$ denotes the family of all continuous $\mathbb{R}^{n}$-valued functions, and the norm $\|\xi\|=\sup _{-\tau \leq s \leq 0}|\xi(s)|$. Denote by $\mathbb{C}_{\mathscr{F}_{0}}^{b}\left([-\tau, 0] ; \mathbb{R}^{n}\right)$ the family of all $\mathscr{F}_{0}$-measurable, bounded and $\mathbb{C}^{b}\left([-\tau, 0] ; \mathbb{R}^{n}\right)$-value random variables. If $x(t)$ is a continuous $\mathbb{R}^{n}$-valued stochastic process on $t \in[-\tau, \infty)$, we let $x_{t}=\{x(t+\theta):-\tau \leq \theta \leq 0\}$ for $t \geq 0$ which is regarded as a $\mathbb{C}\left([-\tau, 0] ; \mathbb{R}^{n}\right)$-valued stochastic process. $\mathbb{C}^{2,1}\left(\mathbb{R}_{+} \times S \times\right.$ $\left.\mathbb{R}^{n} ; \mathbb{R}\right)$ denotes the set of functions from $\mathbb{R}_{+} \times S \times \mathbb{R}^{n}$ to $\mathbb{R}$ which are continuously twice differentiable in $x \in \mathbb{R}^{n}$ and once differentiable in $t \in \mathbb{R}_{+}$.

\section{Problem Formulation and Preliminaries}

Let $\{r(t)\}_{t \geq 0}$ be a right-continuous Markov chain on the probability space taking values in a finite state space $S=$ $\{1,2, \ldots, N\}$ with generator $\Gamma=\left(\gamma_{i j}\right)_{N \times N}$ given by

$$
\mathbb{P}\{r(t+\delta)=j \mid r(t)=i\}= \begin{cases}\gamma_{i j} \delta+o(\delta), & \text { if } i \neq j, \\ 1+\gamma_{i j} \delta+o(\delta), & \text { if } i=j\end{cases}
$$

where $\delta>0$ and $\gamma_{i j} \geq 0$ is the transition rate from $i$ to $j$ if $i \neq j$ while

$$
\gamma_{i i}=-\sum_{j=1, j \neq i}^{N} \gamma_{i j}
$$

Consider the following neutral-type neural networks called drive system and represented by the compact form as follows:

$$
\begin{aligned}
d[x(t)- & D(r(t)) x(t-\tau)] \\
=[- & C(r(t)) x(t)+A(r(t)) f(x(t))+B(r(t)) \\
& \times f(x(t-\tau))+E(r(t)) \\
& \left.\times \int_{t-\tau}^{t} f(x(s)) d s+J(r(t))\right] d t,
\end{aligned}
$$

where $x(t)=\left[x_{1}(t), x_{2}(t), \ldots, x_{n}(t)\right]^{T} \in \mathbb{R}^{n}$ is the state vector associated with $n$ neurons, $f(\cdot)$ denotes the neuron activation functions, and $\tau$ represents the transmission delay. For $t \geq 0$, we denote $r(t)=i, A(r(t))=A^{i}, B(r(t))=B^{i}$, $C(r(t))=C^{i}, D(r(t))=D^{i}, E(r(t))=E^{i}$, and $J(r(t))=$ $J^{i}$, respectively. In neural network (3), $\forall i \in S, A^{i}, B^{i}$, and $E^{i}$ are the connection weight, the discrete delay connection weight, and distributed delay connection weight matrix, respectively; $C^{i}=\operatorname{diag}\left\{c_{1}^{i}, c_{2}^{i}, \ldots, c_{n}^{i}\right\}$ is a positive diagonal matrix; $D^{i}$ is called the neutral-type parameter matrix; $J^{i}=$ $\left[J_{1}^{i}, J_{2}^{i}, \ldots, J_{n}^{i}\right]^{T} \in \mathbb{R}^{n}$ is the constant external input vector. form:

The initial condition of system (3) is given in the following

$$
x(s)=\xi_{x}(s), \quad s \in[-\tau, 0], \quad r(0)=i_{0}
$$

for any $\xi_{x} \in \mathbb{L}_{\mathscr{F}_{0}}^{2}\left([-\tau, 0] ; \mathbb{R}^{n}\right)$.

For the drive system (3), the response system is

$$
\begin{aligned}
d[y(t) & -D(r(t)) y(t-\tau)] \\
=[- & C(r(t)) y(t)+A(r(t)) f(y(t)) \\
& +B(r(t)) f(y(t-\tau))+E(r(t)) \\
& \left.\times \int_{t-\tau}^{t} f(y(s)) d s+J(r(t))+U(r(t))\right] d t \\
& +\sigma(t, r(t), y(t)-x(t), y(t-\tau)-x(t-\tau)) d \omega(t),
\end{aligned}
$$

where $y(t)=\left[y_{1}(t), y_{2}(t), \ldots, y_{n}(t)\right]^{T} \in \mathbb{R}^{n}$ is the state vector of the response system (5), $U(r(t))=U^{i}=$ $\left[U_{1}^{i}, U_{2}^{i}, \ldots, U_{n}^{i}\right]^{T} \in \mathbb{R}^{n}$ is a control input vector, $\omega(t)=$ $\left[\omega_{1}(t), \omega_{2}(t), \ldots, \omega_{n}(t)\right]^{T}$ is an $n$-dimensional Brownian motion defined on the complete probability space $(\Omega, \mathscr{F}, P)$ with a natural filtration $\left\{\mathscr{F}_{t}\right\}_{t \geq 0}$ (i.e., $\mathscr{F}_{t}=\sigma\{\omega(s): 0 \leq s \leq t\}$ is a $\sigma$-algebra) and is independent of the Markovian process $\{r(t)\}_{t \geq 0}$, and $\sigma: \mathbb{R}_{+} \times S \times \mathbb{R}^{n} \times \mathbb{R}^{n} \rightarrow \mathbb{R}^{n \times n}$ is the noise intensity matrix. It is known that external random fluctuation and other probabilistic causes often lead to this type of stochastic perturbations. 

form:

The initial condition of system (5) is given in the following

$$
y(s)=\xi_{y}(s), \quad s \in[-\tau, 0], \quad r(0)=i_{0}
$$

for any $\xi_{y} \in \mathbb{L}_{\mathscr{F}_{0}}^{2}\left([-\tau, 0] ; \mathbb{R}^{n}\right)$.

Let $e(t)=y(t)-x(t)$ be the synchronization error vector. From the drive system and the response system, the error system can be written as follows:

$$
\begin{aligned}
d[e(t) & \left.-D(r(t)) e_{\tau}\right] \\
= & {\left[-C(r(t)) e(t)+A(r(t)) g(e(t))+B(r(t)) g\left(e_{\tau}\right)\right.} \\
& \left.+E(r(t)) \int_{t-\tau}^{t} g(e(s)) d s+U(r(t))\right] d t \\
& +\sigma\left(t, r(t), e(t), e_{\tau}\right) d \omega(t),
\end{aligned}
$$

where $e_{\tau}=e(t-\tau), \quad g(e(t))=f(x(t)+e(t))-f(x(t))$.

The initial condition of system ( 7 ) is given in the following form:

$$
e(s)=\xi(s)=\xi_{y}(s)-\xi_{x}(s), \quad s \in[-\tau, 0], \quad r(0)=i_{0},
$$

with $e(0)=0$.

The primary object here is to deal with the adaptive synchronization problem of the drive system (3) and the response system (5) and derive sufficient conditions such that the response system (5) synchronizes with the drive system (3).

To prove our main results, the following assumptions are needed.

Assumption 1. The activation functions of the neurons $f(\cdot)$ satisfy the Lipschitz condition. That is, there exists a constant $L_{1}, L_{2}>0$ such that

$$
L_{1}|x-y| \leq|f(x)-f(y)| \leq L_{2}|x-y|, \quad \forall x, y \in R^{n} .
$$

Assumption 2. The noise intensity matrix $\sigma(\cdot, \cdot, \cdot, \cdot)$ satisfies the bounded condition. That is, there exist two positive constants $H_{1}$ and $H_{2}$, such that

$$
\begin{aligned}
& \operatorname{trace}\left(\sigma(t, r(t), x(t), y(t))^{T} \sigma(t, r(t), x(t), y(t))\right) \\
& \quad \leq H_{1}|x(t)|^{2}+H_{2}|y(t)|^{2},
\end{aligned}
$$

for all $(t, r(t), x(t), y(t)) \in R_{+} \times S \times R^{n} \times R^{n}$, and $\sigma(t, i, 0,0)=0$ for all $(t, i) \in \mathbb{R}_{+} \times S$.

Assumption 3. For the external input matrix $D^{i}(i \in S)$, there exists positive constant $\kappa_{i} \in(0,1)$, such that

$$
\rho\left(D^{i}\right)=\kappa_{i} \leq \kappa \in(0,1),
$$

where $\kappa=\max _{i \in S} \kappa_{i}$ and $\rho\left(D^{i}\right)$ is the spectral radius of matrix $D^{i}$.
The following concept is necessary in this paper.

Definition 4 (see [21]). The trivial solution $e\left(t ; \xi, i_{0}\right)$ of the error system (7) is said to be almost surely asymptotically stable if

$$
\mathbb{P}\left(\lim _{t \rightarrow \infty}\left|e\left(t ; \xi, i_{0}\right)\right|=0\right)=1
$$

for any initial value $\xi \in \mathbb{C}\left([-\tau, 0] ; \mathbb{R}^{n}\right)$.

If the error system (7) is almost surely asymptotically stable, then the drive system (3) and the response system (5) are said to be almost surely asymptotically synchronization.

Consider the more general neutral-type stochastic delay differential equation (NSDDE) with Markovian switching:

$$
\begin{aligned}
& d[x(t)-\bar{D}(x(t-\tau), r(t))] \\
&= \bar{F}(t, r(t), x(t), x(t-\tau)) d t \\
&+\bar{G}(t, r(t), x(t), x(t-\tau)) d B(t),
\end{aligned}
$$

where $B(t)$ is an $n$-dimensional Brownian motion defined on the probability space $(\Omega, \mathscr{F}, \mathbb{P})$ but independent of the Markov chain $\{r(t)\}_{t \geq 0}$ and

$$
\begin{gathered}
\bar{D}: \mathbb{R}^{n} \times S \longrightarrow \mathbb{R}^{n}, \\
\bar{F}: \mathbb{R}_{+} \times S \times \mathbb{R}^{n} \times \mathbb{R}^{n} \longrightarrow \mathbb{R}^{n}, \\
\bar{G}: \mathbb{R}_{+} \times S \times \mathbb{R}^{n} \times \mathbb{R}^{n} \longrightarrow \mathbb{R}^{n \times n}
\end{gathered}
$$

are all Borel-measurable functions.

For NSDDE (13), the following hypothesis is needed.

(H1) Both $\bar{F}$ and $\bar{G}$ satisfy the local Lipschitz condition. That is, for each $h>0$, there is an $L_{h}>0$ such that

$$
\begin{aligned}
& |\bar{F}(t, i, x, y)-\bar{F}(t, i, \bar{x}, \bar{y})| \\
& \quad \vee|\bar{G}(t, i, x, y)-\bar{G}(t, i, \bar{x}, \bar{y})| \\
& \quad \leq L_{h}(|x-\bar{x}|+|y-\bar{y}|)
\end{aligned}
$$

for all $(t, i) \in \mathbb{R}_{+} \times S$ and those $x, y, \bar{x}, \bar{y} \in \mathbb{R}^{n}$ with $x \vee y \vee$ $\bar{x} \vee \bar{y} \leq h$.

$(\mathrm{H} 1)^{\prime}$ Given any initial data $\{x(\theta):-\tau \leq \theta \leq 0\}=\xi \epsilon$ $\mathbb{C}_{\mathbb{F}_{0}}^{b}\left([-\tau, 0] ; \mathbb{R}^{n}\right),(13)$ has a unique solution denoted by $x\left(t ; \xi, i_{0}\right)$ on $t \geq 0$. Moreover, both $\bar{F}(t, r(t), x(t), y(t))$ and $\bar{G}(t, r(t), x(t), y(t))$ are locally bounded in $(x, y)$ while uniformly bounded in $(t, r(t))$. That is, for any $h>0$, there is a $K_{h}>0$, such that

$$
|\bar{F}(t, r(t), x(t), y(t))| \vee|\bar{G}(t, r(t), x(t), y(t))| \leq K_{h},
$$

for all $t \geq 0, r(t) \in S$, and $x, y \in \mathbb{R}^{n}$ with $|x| \vee|y| \leq h$. 
(H2) For each $i \in S$, there is a constant $\kappa_{i} \in(0,1)$ such that

$$
|\bar{D}(x, i)-\bar{D}(\bar{x}, i)| \leq \kappa_{i}|x-\bar{x}| \quad \forall x, \bar{x} \in \mathbb{R}^{n} .
$$

(H3) For each $(t, i) \in \mathbb{R}_{+} \times S$,

$$
\bar{F}(t, i, 0,0)=0, \quad \bar{G}(t, i, 0,0)=0, \quad \bar{D}(0, i)=0 .
$$

Then, we present some preliminary lemmas which play an important role in the proof of the main results.

Lemma 5 (see [16]). Let $x, y \in \mathbb{R}^{n}$. Then

$$
x^{T} y+y^{T} x \leq \epsilon x^{T} x+\epsilon^{-1} y^{T} y
$$

for any $\epsilon>0$.

Lemma 6 (the generalized Ito formula, see [17]). Let $V \in$ $\mathbb{C}^{2,1}\left(\mathbb{R}_{+} \times S \times \mathbb{R}^{n} ; \mathbb{R}\right)$ and $x(t)$ be a solution of neutral-type stochastic delay differential equation (13).

Then for any stopping times $0 \leq \rho_{1} \leq \rho_{2}<\infty$ a.s.

$$
\begin{aligned}
& \mathbb{E} V\left(\rho_{2}, r\left(\rho_{2}\right), x\left(\rho_{2}\right)-\bar{D}\left(x\left(\rho_{2}-\tau\right), r\left(\rho_{2}\right)\right)\right) \\
&= \mathbb{E} V\left(\rho_{1}, r\left(\rho_{1}\right), x\left(\rho_{1}\right)-\bar{D}\left(x\left(\rho_{1}-\tau\right), r\left(\rho_{1}\right)\right)\right) \\
& \quad+\mathbb{E} \int_{\rho_{1}}^{\rho_{2}} \mathscr{L} V(s, r(s), x(s), x(s-\tau)) d s
\end{aligned}
$$

holds provided that $V(t, r(t), x(t)-\bar{D}(x(t), r(t)))$ and $\mathscr{L} V(t, r(t), x(t), x(t-\tau))$ are bounded on $t \in\left[\rho_{1}, \rho_{2}\right]$ with probability 1 , where the operator $\mathscr{L} V: \mathbb{R}_{+} \times S \times \mathbb{R}^{n} \times \mathbb{R}^{n} \rightarrow \mathbb{R}$ is defined by

$$
\begin{aligned}
\mathscr{L} V & (t, i, x, y) \\
= & V_{t}(t, i, x-\bar{D}(y, i))+V_{x}(t, i, x-\bar{D}(y, i)) \bar{F}(t, i, x, y) \\
& +\frac{1}{2} \operatorname{trace}\left[\bar{G}^{T}(t, i, x, y) V_{x x}(t, i, x-\bar{D}(y, i))\right. \\
& \times \bar{G}(t, i, x, y)] \\
& +\sum_{j=1}^{N} \gamma_{i j} V(t, j, x-\bar{D}(y, i)), \\
V_{t}(t, i, x(t)) & \frac{\partial V(t, i, x(t))}{\partial t}, \\
= & V_{x}(t, i, x(t)) \\
= & \left(\frac{\partial V(t, i, x(t))}{\partial x_{1}}, \frac{\partial V(t, i, x(t))}{\partial x_{2}}, \ldots, \frac{\partial V(t, i, x(t))}{\partial x_{n}}\right), \\
V_{x x}(t, i, x(t)) & \left(\frac{\partial^{2} V(t, i, x(t))}{\partial x_{j} \partial x_{k}}\right)_{n \times n} .
\end{aligned}
$$

Now we cite the convergence theorem of nonnegative semimartingales (see [22], Theorem 7 on page 139) which is a useful lemma.

Lemma 7 (the convergence theorem of nonnegative semimartingales). Let $A_{1}(t)$ and $A_{2}(t)$ be two continuous adapted increasing processes on $t \geq 0$ with $A_{1}(0)=A_{2}(0)=0$ a.s. Let $M(t)$ be a real-valued continuous local martingale with $M(0)=0$ a.s. Let $\zeta$ be a nonnegative $\mathscr{F}_{0}$-measurable random variable such that $\mathbb{E} \zeta<\infty$. Define

$$
X(t)=\zeta+A_{1}(t)-A_{2}(t)+M(t), \quad t \geq 0 .
$$

If $X(t)$ is nonnegative, then

$$
\begin{gathered}
\left\{\lim _{t \rightarrow \infty} A_{1}(t)<\infty\right\} \subset\left\{\lim _{t \rightarrow \infty} X(t)<\infty\right\} \\
\cap\left\{\lim _{t \rightarrow \infty} A_{2}(t)<\infty\right\} \text { a.s. }
\end{gathered}
$$

where $C \subset D$ a.s. means $\mathbb{P}\left(C \cap D^{c}\right)=0$. In particular, if $\lim _{t \rightarrow \infty} A_{1}(t)<\infty$ a.s., then, with probability one, we have

$$
\begin{gathered}
\lim _{t \rightarrow \infty} X(t)<\infty, \quad \lim _{t \rightarrow \infty} A_{2}(t)<\infty, \\
-\infty<\lim _{t \rightarrow \infty} M(t)<\infty .
\end{gathered}
$$

That is, all of the three processes $X(t), A_{2}(t)$, and $M(t)$ converge to finite random variables.

Lemma 8 (Hölder inequality; see [21]). Let $a_{i} \in \mathbb{R}, k, p \in \mathbb{Z}$, and $p \geq 1$. Then

$$
\left|\sum_{i=1}^{k} a_{i}\right|^{p} \leq k^{p-1} \sum_{i=1}^{k}\left|a_{i}\right|^{p}
$$

Lemma 9 (Doob martingale inequality; see [21]). Let $\left\{M_{t}\right\}_{t \geq 0}$ be an $\mathbb{R}^{n}$-martingale. Let $[a, b]$ be a bounded interval in $\mathbb{R}_{+}$. If $p>1$, and $M_{t} \in \mathbb{L}^{p}\left(\Omega ; \mathbb{R}^{n}\right)$ (the family of $\mathbb{R}^{n}$-valued random variables $X$ with $\left.\mathbb{E}|X|^{p}<\infty\right)$, then

$$
\mathbb{E}\left(\sup _{a \leq t \leq b}\left|M_{t}\right|^{p}\right) \leq\left(\frac{p}{p-1}\right)^{p} \mathbb{E}\left|M_{b}\right|^{p} .
$$

Lemma 10 (Chebyshev's inequality; see [21]). If $c>0, p>$ $0, X \in \mathbb{L}^{p}\left(\Omega ; \mathbb{R}^{n}\right)$, then

$$
\mathbb{P}\{\omega:|X(\omega)| \geq c\} \leq c^{-p} \mathbb{E}|X|^{p} .
$$

\section{Main Results}

In this section, we give some criteria of adaptive synchronization for the drive system (3) and the response system (5). First, we establish a general result which can be applied widely.

\subsection{Almost Surely Asymptotically Stable}

Theorem 11. Let (H1), (H2), and (H3) hold. Assume that there are functions $V \in \mathbb{C}^{2,1}\left(\mathbb{R}_{+} \times S \times \mathbb{R}^{n} ; \mathbb{R}\right), \gamma \in \mathbb{L}^{1}\left(\mathbb{R}_{+} ; \mathbb{R}_{+}\right)$, and $W_{1}, W_{2}, W_{3} \in \mathbb{C}\left(\mathbb{R}^{n} ; \mathbb{R}_{+}\right)$such that 
(C1)

$$
|x| \longrightarrow \infty, \quad i \in S, 0 \leq t<\infty
$$

(C2)

$$
\begin{aligned}
& \mathscr{L} V(t, i, x, y) \\
& \quad \leq \gamma(t)-W_{1}(x)+W_{2}(y)-W_{3}(x-D(y, i)),
\end{aligned}
$$

for $(t, i, x, y) \in \mathbb{R}_{+} \times S \times \mathbb{R}^{n} \times \mathbb{R}^{n}$, and

$$
\lim _{|x| \rightarrow \infty}\left[\inf _{(t, i) \in \mathbb{R}_{+} \times S} V(t, i, x)\right]=\infty,
$$

(C3)

$$
\begin{gathered}
W_{1}(0)=W_{2}(0)=W_{3}(0)=0, \\
W_{1}(x) \geq W_{2}(x), \quad \forall x \neq 0 .
\end{gathered}
$$

Then for any initial data $\{x(\theta):-\bar{\tau} \leq \theta \leq 0\}=\xi \epsilon$ $\mathbb{C}_{\mathscr{F}_{0}}^{b}\left([-\bar{\tau}, 0] ; \mathbb{R}^{n}\right)$ and $r(0)=i_{0} \in S$, one has the following.

(R1) Equation (13) has a unique global solution which is denoted by $x\left(t ; \xi, i_{0}\right)$.

(R2) Assume that $W_{3}(x)=0$ if and only if $x=0$. The solution $x\left(t ; \xi, i_{0}\right)$ obeys that

$$
\lim _{t \rightarrow \infty} x\left(t ; \xi, i_{0}\right)=0 \quad \text { a.s. }
$$

That is, $x\left(t ; \xi, i_{0}\right)$ is almost surely asymptotically stable.

The proof of this theorem is given in the Appendix.

Remark 12. Theorem 11 is an extension of Theorem 11 in [16]; that is, when we take $W_{1}(x)=W_{2}(x)$ in our theorem, then Theorem 11 is coincident with Theorem 3.1 in [16]. Moreover, Theorem 11 is also an extension of Theorem 2.1 in [23] when we take $W_{3}(x)=0$ with $\bar{D}=0$.

Remark 13. From the proof of Theorem 11, we can see that if condition (H1) is substituted by $(\mathrm{H} 1)^{\prime}$, then the conclusion (R2) is also true.

3.2. Almost Sure Asymptotical Synchronization. In this subsection, we give a criterion of adaptive almost sure asymptotical synchronization for the drive system (3) and the response system (5).

Theorem 14. For systems (3) and (5), let Assumptions 1-3 hold, and the error system (7) has a unique solution denoted by $e\left(t ; \xi, i_{0}\right)$ on $t \geq 0$ for any initial data $\{e(\theta):-\tau \leq \theta \leq 0\}=$ $\xi \in \mathbb{C}_{\mathbb{E}_{0}}^{b}\left([-\tau, 0] ; \mathbb{R}^{n}\right)$ with $e(0)=0$.
Assume also that there exist symmetric matrix $Q_{1}>0$, diagonal matrix $P^{i}>0(i=1, \ldots, N)$, and positive scalars $\rho, \rho_{1}, \rho_{2}, \epsilon_{i}(i=1,2,3,4)$, such that

$$
\begin{aligned}
& \rho_{2} I<Q_{1}<\rho_{1} I, \\
& P^{i}<\rho I, \\
& {\left[\begin{array}{cccc}
\left(L_{2}^{2} \rho_{1}+H_{1} \rho\right) I-2 P^{i} C^{i} & C^{i} P^{i} & L_{2} I & \tau L_{2} I \\
* & -\epsilon_{1} I & 0 & 0 \\
* & 0 & -\epsilon_{2} I & 0 \\
* & 0 & 0 & -\epsilon_{4} I
\end{array}\right]<0,} \\
& \left(\rho_{2} L_{1}^{2}-\rho H_{2}-\epsilon_{3}^{-1} L_{2}^{2}\right) I-\epsilon_{1} D^{i T} D^{i}<0, \\
& {\left[\begin{array}{cccc}
\sum_{k=1}^{N} \gamma_{i k} P^{k}+2 P^{i} K^{*} & \epsilon_{2} P^{i} A^{i} & \epsilon_{3} P^{i} B^{i} & \epsilon_{4} P^{i} E^{i} \\
* & -\epsilon_{2} I & 0 & 0 \\
* & 0 & -\epsilon_{3} I & 0 \\
* & 0 & 0 & -\epsilon_{4} I
\end{array}\right]<0,} \\
& {\left[\begin{array}{ccccc}
\Xi_{11} & C^{i} P^{i} & L_{2} I & L_{2} I & \tau L_{2} I \\
* & -\epsilon_{1} I & 0 & 0 & 0 \\
* & 0 & -\epsilon_{2} I & 0 & 0 \\
* & 0 & 0 & -\epsilon_{3} I & 0 \\
* & 0 & 0 & 0 & -\epsilon_{4} I
\end{array}\right]<0,}
\end{aligned}
$$

where $i=1,2, \ldots, N, K^{*}=\operatorname{diag}\left\{k_{1}^{*}, k_{2}^{*}, \ldots, k_{n}^{*}\right\}$ with $k_{j}^{*}$ being arbitrary negative constants to be chosen, and $\Xi_{11}=\left(L_{2}^{2} \rho_{1}+\right.$ $\left.H_{1} \rho-\rho_{2} L_{1}^{2}+H_{2} \rho\right) I-2 P^{i} C^{i}+\epsilon_{1} D^{i T} D^{i}$.

We choose the feedback control $U^{i}$ with the update law as $U^{i}=\operatorname{diag}\left\{k_{1}, \ldots, k_{n}\right\}\left(e-D^{i} e_{\tau}\right)$ and $\dot{k}_{j}=-\beta_{j} p_{j}^{i}\left(e-D^{i}\left(e_{\tau}\right)\right)_{j}^{2}$, where $\beta_{j}>0(j=1,2, \ldots, n)$ are arbitrary constants, $p_{j}^{i}$ is the $j$ th diagonal entry of matrix $P^{i}$, and $\left(e-D^{i} e_{\tau}\right)_{j}$ is the $j$ th element of $e-D^{i} e_{\tau}$. Then the error system (7) is almost surely asymptotically stable. Therefore, the drive system (3) and the response system (5) are adaptive synchronized a.s.

Proof. Under Assumptions 1-3 and the existence of $e\left(t ; \xi, i_{0}\right)$, it can be seen that $\bar{F}\left(t, r(t), e(t), e_{\tau}(t)\right), \bar{G}\left(t, r(t), e(t), e_{\tau}(t)\right)$, and $\bar{D}\left(e_{\tau}(t), r(t)\right)$ satisfy $(\mathrm{H} 1)^{\prime},(\mathrm{H} 2)$, and (H3), where

$$
\begin{gathered}
\bar{F}\left(t, r(t), e(t), e_{\tau}(t)\right) \\
=-C(r(t)) e(t)+A(r(t)) g(e(t))+B(r(t)) g\left(e_{\tau}(t)\right) \\
+E(r(t)) \int_{t-\tau}^{t} g(e(s)) d s+U(r(t)), \\
\bar{G}\left(t, r(t), e(t), e_{\tau}(t)\right)=\sigma\left(t, r(t), e(t), e_{\tau}(t)\right), \\
\bar{D}\left(e_{\tau}(t), r(t)\right)=D(r(t)) e_{\tau}(t) .
\end{gathered}
$$


For each $i \in S$, choosing a nonnegative function

$$
\begin{aligned}
V(t, i, e)= & x^{T} P^{i} x+\int_{t-\tau}^{t} g^{T}(e(s)) Q_{1} g(e(s)) d s \\
& +\int_{-\tau}^{0} \int_{t+s}^{t} e^{T}(\theta) Q_{2} e(\theta) d \theta d s \\
& +\sum_{j=1}^{n} \frac{1}{\beta_{j}}\left(k_{j}-k_{j}^{*}\right)^{2},
\end{aligned}
$$

where $Q_{2}=\varepsilon_{4}^{-1} \tau L_{2}^{2} I$, and computing $\mathscr{L} V\left(t, i, e, e_{\tau}\right)$ along the trajectory of error system (7), we have

$$
\begin{aligned}
& \mathscr{L V}\left(t, i, e, e_{\tau}\right) \\
& =V_{t}\left(t, i, e-D^{i} e_{\tau}\right) \\
& +V_{x}\left(t, i, e-D^{i} e_{\tau}\right)\left[-C^{i} e+A^{i} g(e)+B^{i} g\left(e_{\tau}\right)\right. \\
& \left.\quad+E^{i} \int_{t-\tau}^{t} g(e(s)) d s+U^{i}\right] \\
& +\frac{1}{2} \operatorname{trace}\left[\sigma^{T}\left(t, i, e, e_{\tau}\right) V_{x x}\left(t, i, e-D^{i} e_{\tau}\right) \sigma\left(t, i, e, e_{\tau}\right)\right] \\
& +\sum_{k=1}^{N} \gamma_{i k} V\left(t, k, e-D^{i} e_{\tau}\right) .
\end{aligned}
$$

While

$$
\begin{aligned}
V_{t}(t, & \left.i, e-D^{i} e_{\tau}\right) \\
= & g^{T}(e(t)) Q_{1} g(e(t))-g^{T}(e(t-\tau)) Q_{1} g(e(t-\tau)) \\
& +\tau e^{T}(t) Q_{2} e(t)-\int_{-\tau}^{0} e^{T}(t+s) Q_{2} e(t+s) d s \\
& +\sum_{j=1}^{n} \frac{2}{\beta_{j}}\left(k_{j}-k_{j}^{*}\right) \dot{k}_{j} \\
= & g^{T}(e(t)) Q_{1} g(e(t))-g^{T}\left(e_{\tau}\right) Q_{1} g\left(e_{\tau}\right) \\
& +\tau e^{T}(t) Q_{2} e(t)-\int_{t-\tau}^{t} e^{T}(s) Q_{2} e(s) d s \\
& -2 \sum_{j=1}^{n}\left(k_{j}+k_{j}^{*}\right) p_{j}^{i}\left(e-D^{i} e_{\tau}\right)_{j}^{2} \\
& V_{x}\left(t, i, e-D^{i} e_{\tau}\right)=2\left(e-D^{i} e_{\tau}\right)^{T} P^{i} \\
& V_{x x}\left(t, i, e-D^{i} e_{\tau}\right)=2 P^{i} \\
& \sum_{k=1}^{N} \gamma_{i k} V\left(t, k, e-D^{i} e_{\tau}\right) \\
& \sum_{k=1}^{N} \gamma_{i k}\left(e-D^{i} e_{\tau}\right)^{T} P^{k}\left(e-D^{i} e_{\tau}\right)
\end{aligned}
$$

so

$$
\begin{aligned}
& \mathscr{L} V\left(t, i, e, e_{\tau}\right) \\
& \leq g^{T}(e) Q_{1} g(e)-g^{T}\left(e_{\tau}\right) Q_{1} g\left(e_{\tau}\right)+\tau e^{T} Q_{2} e \\
& \quad-\int_{t-\tau}^{t} e^{T}(s) Q_{2} e(s) d s-2 \sum_{j=1}^{n}\left(k_{j}-k_{j}^{*}\right) \\
& \times p_{j}^{i}\left(e-D^{i} e_{\tau}\right)_{j}^{2}+2\left(e-D^{i} e_{\tau}\right)^{T} \\
& \times P^{i}\left[-C^{i} e+A^{i} g(e)+B^{i} g\left(e_{\tau}\right)\right. \\
& \quad+E^{i} \int_{t-\tau}^{t} g(e(s)) d s \\
& \left.\quad+\operatorname{diag}\left\{k_{1}, \ldots, k_{n}\right\}\left(e-D^{i} e_{\tau}\right)\right] \\
& +\operatorname{trace}\left[\sigma^{T}\left(t, i, e, e_{\tau}\right) P^{i} \sigma\left(t, i, e, e_{\tau}\right)\right] \\
& +\sum_{k=1}^{N} \gamma_{i k}\left(e-D^{i} e_{\tau}\right)^{T} P^{k}\left(e-D^{i} e_{\tau}\right) .
\end{aligned}
$$

It is easy to get that

$$
\begin{aligned}
& 2\left(e-D^{i} e_{\tau}\right)^{T} P^{i} \operatorname{diag}\left\{k_{1}, \ldots, k_{n}\right\}\left(e-D^{i} e_{\tau}\right) \\
& =2 \sum_{j=1}^{n} k_{j} p_{j}^{i}\left(e-D^{i} e_{\tau}\right)_{j}^{2} .
\end{aligned}
$$

By (44), we have

$$
\begin{aligned}
& 2\left(e-D^{i} e_{\tau}\right)^{T} P^{i} \operatorname{diag}\left\{k_{1}, \ldots, k_{n}\right\}\left(e-D^{i} e_{\tau}\right) \\
& \quad-2 \sum_{j=1}^{n}\left(k_{j}-k_{j}^{*}\right) p_{j}^{i}\left(e-D^{i} e_{\tau}\right)_{j}^{2} \\
& =2 \sum_{j=1}^{n} k_{j}^{*} p_{j}^{i}\left(e-D^{i} e_{\tau}\right)_{j}^{2} \\
& =2\left(e-D^{i} e_{\tau}\right)^{T} P^{i} K^{*}\left(e-D^{i} e_{\tau}\right) .
\end{aligned}
$$

According to Assumption 1 and Lemma 5, we have that

$$
\begin{gathered}
g^{T}(e) Q_{1} g(e) \leq \lambda_{\max }\left(Q_{1}\right) g^{T}(e) g(e) \leq \rho_{1} L_{2}^{2}|e|^{2}, \\
-g^{T}\left(e_{\tau}\right) Q_{1} g\left(e_{\tau}\right) \\
\leq-\lambda_{\min }\left(Q_{1}\right) g^{T}\left(e_{\tau}\right) g\left(e_{\tau}\right) \leq-\rho_{2} L_{1}^{2}\left|e_{\tau}\right|^{2}, \\
2 e_{\tau}^{T} D^{i T} P^{i} C^{i} e \leq \epsilon_{1} e_{\tau}^{T} D^{i T} D^{i} e_{\tau}+\epsilon_{1}^{-1} e^{T} C^{i} P^{i} P^{i} C^{i} e, \\
2\left(e-D^{i} e_{\tau}\right)^{T} P^{i} A^{i} g(e) \\
\leq \epsilon_{2}\left(e-D^{i} e_{\tau}\right)^{T} P^{i} A^{i} A^{i T} P^{i}\left(e-D^{i} e_{\tau}\right)+\epsilon_{2}^{-1} g^{T}(e) g(e) \\
\leq \epsilon_{2}\left(e-D^{i} e_{\tau}\right)^{T} P^{i} A^{i} A^{i T} P^{i}\left(e-D^{i} e_{\tau}\right)+\epsilon_{2}^{-1} L_{2}^{2} e^{T} e,
\end{gathered}
$$




$$
\begin{aligned}
& 2\left(e-D^{i} e_{\tau}\right)^{T} P^{i} B^{i} g\left(e_{\tau}\right) \\
& \leq \epsilon_{3}\left(e-D^{i} e_{\tau}\right)^{T} P^{i} B^{i} B^{i T} P^{i}\left(e-D^{i} e_{\tau}\right)+\epsilon_{3}^{-1} g^{T}(e) g\left(e_{\tau}\right) \\
& \leq \epsilon_{3}\left(e-D^{i} e_{\tau}\right)^{T} P^{i} B^{i} B^{i T} P^{i}\left(e-D^{i} e_{\tau}\right)+\epsilon_{3}^{-1} L_{2}^{2} e_{\tau}^{T} e_{\tau}, \\
& 2\left(e-D^{i} e_{\tau}\right)^{T} P^{i} E^{i} \int_{t-\tau}^{t} g(e(s)) d s \\
& \leq \epsilon_{4}\left(e-D^{i} e_{\tau}\right)^{T} P^{i} E^{i} E^{i T} P^{i}\left(e-D^{i} e_{\tau}\right) \\
&+\epsilon_{4}^{-1}\left(\int_{t-\tau}^{t} g(e(s)) d s\right)^{T}\left(\int_{t-\tau}^{t} g(e(s)) d s\right) \\
& \leq \epsilon_{4}\left(e-D^{i} e_{\tau}\right)^{T} P^{i} E^{i} E^{i T} P^{i}\left(e-D^{i} e_{\tau}\right) \\
&+\epsilon_{4}^{-1} \tau \int_{t-\tau}^{t} g^{T}(e(s)) g(e(s)) d s \\
& \leq \epsilon_{4}\left(e-D^{i} e_{\tau}\right)^{T} P^{i} E^{i} E^{i T} P^{i}\left(e-D^{i} e_{\tau}\right) \\
&+\epsilon_{4}^{-1} \tau L_{2}^{2} \int_{t-\tau}^{t} e^{T}(s) e(s) d s, \\
& \quad \operatorname{trace}\left(\sigma^{T}\left(t, i, e, e_{\tau}\right) P^{i} \sigma\left(t, i, e, e_{\tau}\right)\right) \\
& \leq \rho\left(H_{1} e^{T} e+H_{2} e_{\tau}^{T} e_{\tau}\right) .
\end{aligned}
$$

Substituting (45)-(46) into (43) yields

$$
\begin{gathered}
\mathscr{L} V\left(t, i, e, e_{\tau}\right) \\
\leq e^{T}\left[\rho_{1} L_{2}^{2} I-2 P^{i} C^{i}+\epsilon_{1}^{-1} C^{i T} P^{i} P^{i} C^{i}\right. \\
\left.+\epsilon_{2}^{-1} L_{2}^{2} I+\rho H_{1} I+\varepsilon_{4}^{-1} \tau^{2} L_{2}^{2} I\right] e \\
+e_{\tau}^{T}\left[-\rho_{2} L_{1}^{2} I+\epsilon_{1} D^{i T} D^{i}+\epsilon_{3}^{-1} L_{2}^{2} I\right. \\
\left.+\rho H_{2} I\right] e_{\tau}-\left(e-D^{i} e_{\tau}\right)^{T} \\
\times\left[\epsilon_{2} P^{i} A^{i} A^{i T} P^{i}+\epsilon_{3} P^{i} B^{i} B^{i T} P^{i}+\epsilon_{4} P^{i} E^{i} E^{i T} P^{i}\right. \\
\left.+\sum_{k=1}^{N} \gamma_{i k} P^{k}+2 P^{i} K^{*}\right]\left(e-D^{i} e_{\tau}\right) .
\end{gathered}
$$

Therefore,

$$
\begin{aligned}
& \mathscr{L} V\left(t, i, e, e_{\tau}\right) \\
& \quad \leq-W_{1}(e)+W_{2}\left(e_{\tau}\right)-W_{3}\left(e-D^{i} e_{\tau}\right),
\end{aligned}
$$

where

$$
\begin{aligned}
W_{1}(e) & =e^{T} \bar{W}_{1} e, \\
W_{2}\left(e_{\tau}\right) & =e_{\tau}^{T} \bar{W}_{2} e_{\tau}, \\
W_{3}\left(e-D^{i} e_{\tau}\right) & =\left(e-D^{i} e_{\tau}\right)^{T} \bar{W}_{3}\left(e-D^{i} e_{\tau}\right),
\end{aligned}
$$

with

$$
\begin{gathered}
\bar{W}_{1}=-\left[\rho_{1} L_{2}^{2} I-2 P^{i} C^{i}+\epsilon_{1}^{-1} C^{i T} P^{i} P^{i} C^{i}\right. \\
\left.+\epsilon_{2}^{-1} L_{2}^{2} I+\rho H_{1} I+\varepsilon_{4}^{-1} \tau^{2} L_{2}^{2} I\right] \\
\bar{W}_{2}=-\rho_{2} L_{1}^{2} I+\epsilon_{1} D^{i T} D^{i}+\epsilon_{3}^{-1} L_{2}^{2} I+\rho H_{2} I \\
\overline{W_{3}}=-\left[\epsilon_{2} P^{i} A^{i} A^{i T} P^{i}+\epsilon_{3} P^{i} B^{i} B^{i T} P^{i}\right. \\
\left.+\epsilon_{4} P^{i} E^{i} E^{i T} P^{i}+\sum_{k=1}^{N} \gamma_{i k} P^{k}+2 P^{i} K^{*}\right]
\end{gathered}
$$

Now, (35) is equivalent to $\bar{W}_{1}>0,(36)$ is just $\bar{W}_{2}>$ 0 , (37) is equivalent to $\bar{W}_{3}>0$, and (38) is equivalent to $\bar{W}_{1}>\bar{W}_{2}$. So from the conditions of this theorem, we know that conditions (C1), (C2), and (C3) in Theorem 11 are all satisfied. So by Theorem 11, the error system (7) is almost surely asymptotically stable. And hence the drive system (3) and the response system (5) are adaptive synchronized a.s. The proof of Theorem 14 is completed.

Remark 15. In this section, a numerical example will be given to support the main results obtained in this paper.

\section{Numerical Examples}

In this section, a numerical example will be given to support the main results obtained in this paper.

Letting $\Gamma=\left[\begin{array}{cc}-4 & 4 \\ 2 & -2\end{array}\right]$, which means $N=2$, we give the parameters concerning the drive system (3), the response system (5), and the error system (7) as follows:

$$
\begin{aligned}
& D(1)=\left[\begin{array}{cc}
0.2 & 0 \\
0 & 0.3
\end{array}\right], \quad D(2)=\left[\begin{array}{cc}
0.3 & 0 \\
0 & 0.1
\end{array}\right], \\
& C(1)=\left[\begin{array}{ll}
6 & 1 \\
1 & 7
\end{array}\right], \quad C(2)=\left[\begin{array}{ll}
4 & 0 \\
0 & 7
\end{array}\right], \\
& A(1)=\left[\begin{array}{cc}
-4 & 2 \\
-6 & 2
\end{array}\right], \quad A(2)=\left[\begin{array}{ll}
-3 & 2 \\
-3 & 1
\end{array}\right], \\
& B(1)=\left[\begin{array}{cc}
-2 & 1 \\
1 & -3
\end{array}\right], \quad B(2)=\left[\begin{array}{cc}
-4 & 3 \\
1 & -2
\end{array}\right], \\
& E(1)=\left[\begin{array}{cc}
-5 & 2 \\
2 & -3
\end{array}\right], \quad E(2)=\left[\begin{array}{ll}
-4 & -2 \\
-2 & -3
\end{array}\right], \\
& J(1)=[1,0]^{T}, \quad J(2)=[-1,1]^{T} .
\end{aligned}
$$

We further set $\tau=1, f(\cdot)=\tanh (\cdot), \sigma(\cdot)=e(t)+e_{\tau}(t)$. Then we can confirm that Assumptions 1-3 are satisfied with $L_{1}=0$, $L_{2}=1, H_{1}=H_{2}=2$, and $\kappa_{1}=\kappa_{2}=\kappa=0.3$. 


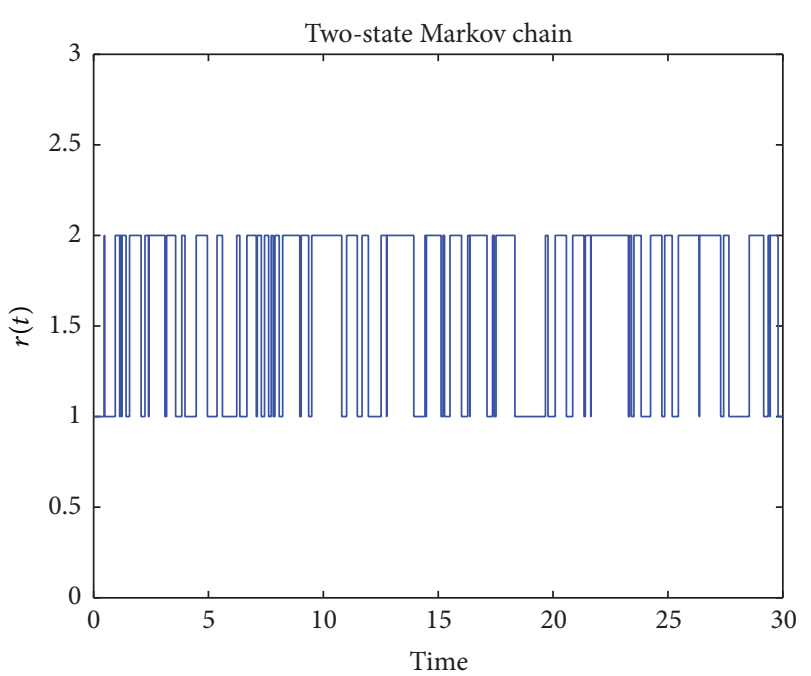

Figure 1: The varying curve of Markov chain with 2 states.

Letting $K^{*}=\left[\begin{array}{ll}6 & 0 \\ 0 & 8\end{array}\right]$ and using LMI toolbox in Matlab, we solve matrix inequalities (33)-(38) and obtain the following results:

$$
\begin{aligned}
& Q_{1}=\left[\begin{array}{cc}
0.3196 & 0 \\
0 & 0.3196
\end{array}\right], \quad P^{1}=\left[\begin{array}{cc}
0.3899 & 0 \\
0 & 0.3899
\end{array}\right], \\
& P^{2}=\left[\begin{array}{cc}
0.4690 & 0 \\
0 & 0.4690
\end{array}\right], \quad \rho=0.5077, \\
& \rho_{1}=0.4498, \quad \rho_{2}=0.1078, \quad \epsilon_{1}=9.9260, \\
& \epsilon_{2}=178.9801, \quad \epsilon_{3}=194.4959, \quad \epsilon_{4}=226.2334 .
\end{aligned}
$$

So from Theorem 14, the drive system (3) and the response system (5) are adaptive synchronized a.s. when the error system (7) has a unique solution.

To illustrate the effectiveness of the result in this paper, we depict the evolution figures of the systems as Figures 1, 2, 3, and 4. Figure 1 shows the two-state Markov chain in the systems. Figure 2 shows that the drive system (3) synchronizes the response system (5) from the moment of $t=7$. It can be seen from Figure 3 that the state of the error system (7) tends to zero from $t=7$, which also describes the synchronization of the drive system (3) and the response system (5). The update law of the adaptive control gain $K(t)$ is depicted in Figure 4. Figure 4 shows us that the update law of the control gain $K(t)$ no longer varies after the response system (5) synchronizes with the drive system (3).

\section{Conclusions}

In this paper, we have proposed a new criterion of a.s. asymptotic stability for a general neutral-type stochastic differential equation which extends the existing results. Based upon this new stability criterion, we have obtained a condition of a.s. asymptotic adaptive synchronization for neutral-type neural networks with stochastic perturbation and Markovian
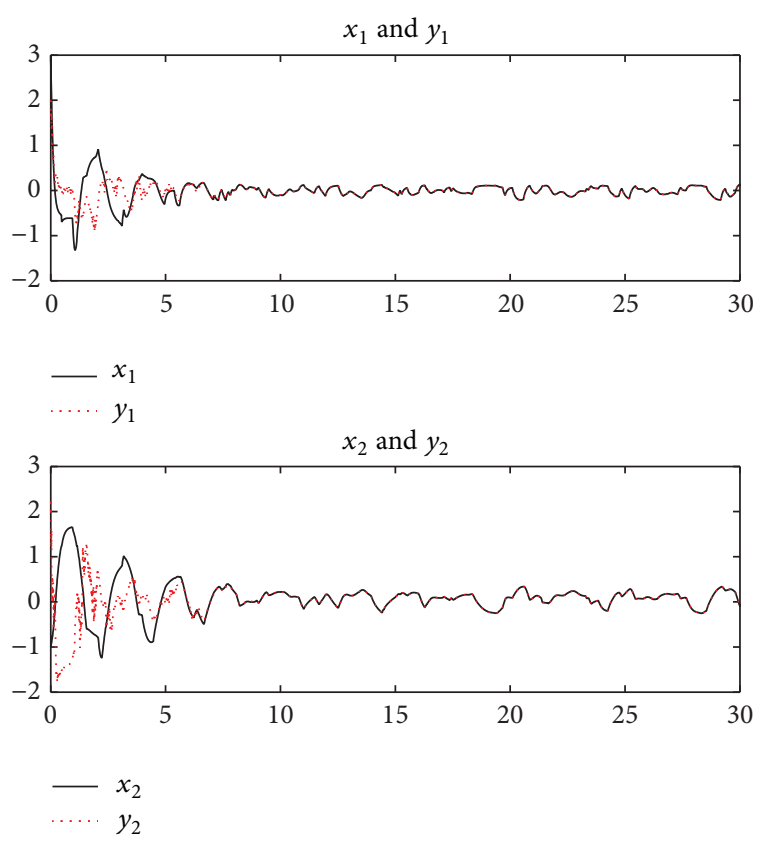

Figure 2: The dynamic trajectory of the drive system and the response system.

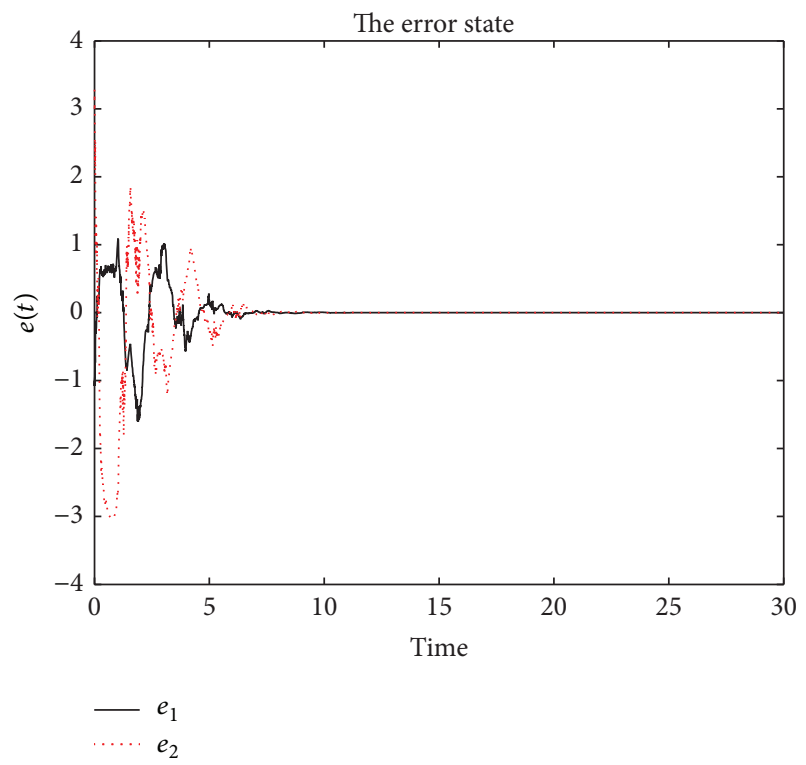

FIGURE 3: The trajectory of the error state.

switching by making use of Lyapunov functional method and designing an adaptive controller. The synchronization condition is expressed as linear matrix inequality which can be easily solved by Matlab. Finally, we have employed a numerical example to illustrate the effectiveness of the method and result obtained in this paper. In the future, we will consider the condition of a.s. asymptotic adaptive synchronization for neutral-type neural networks with timevarying delay by making use of M-matrix method. 


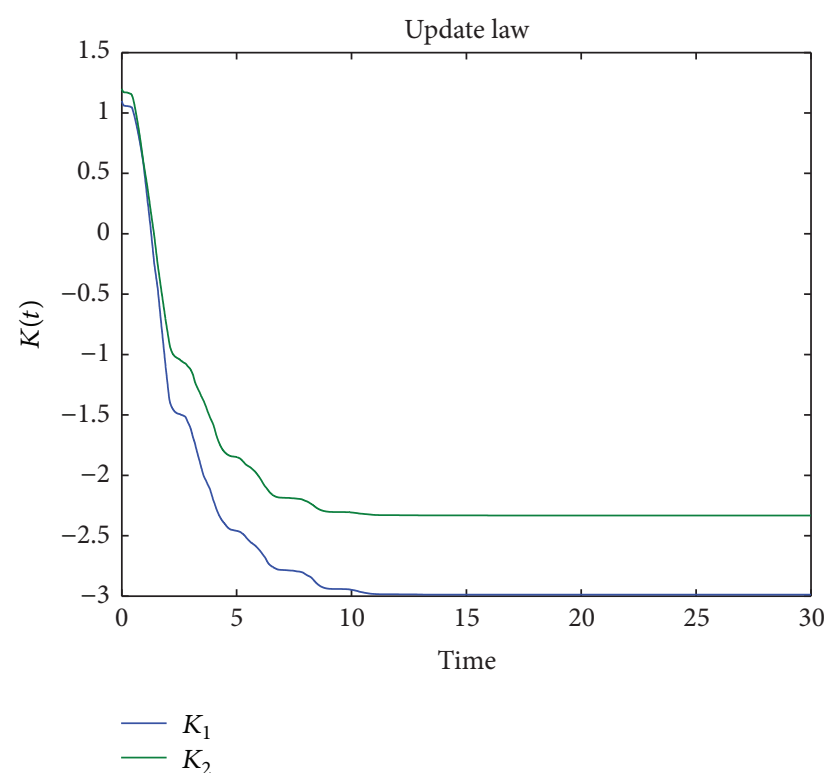

FIGURE 4: The dynamic curve of the update law of the gain $K(t)$.

\section{Appendix}

Proof. The proof of (R1) is the same as [16] and is omitted here. To prove (R2), we will divide it into five steps. We change $\bar{D}$ into $D$ in subsequence for simplicity.

Step 1 . We prove that the solution $x\left(t, i_{0}, \xi\right)$ of the system obeys

$$
\underbrace{\lim \sup }_{t \rightarrow \infty} V(t, r(t), x(t)-D(r(t), x(t-\tau)))<\infty \text { a.s. }
$$

In fact, let

$M(t)$

$$
\begin{gathered}
=\int_{0}^{t} V_{x}(s, r(s), x(s)-D(x(s-\tau), r(s))) d B(s) \\
+\int_{0}^{t} \int_{R}\left(V \left(s, i_{0}+\bar{h}(r(s-), l), x(s)\right.\right. \\
-D(x(s-\tau), r(s))) \\
-V(s, r(s), x(s)-D(x(s-\tau), r(s)))) \mu(d s, d l)
\end{gathered}
$$

which is a continuous local martingale with $M(0)=0$, a.s. By generalized Ito formula (Lemma 6), we have

$$
\begin{aligned}
& V(t, i, x(t)-D(i, x(t-\tau))) \\
& \quad \leq V\left(0, i_{0}, x(0)-D(i, x(-\tau))\right)
\end{aligned}
$$

$$
\begin{aligned}
& +\int_{0}^{t} \mathscr{L} V(s, r(s), x(s), x(s)-D(r(s), x(s-\tau))) d s \\
& +M(t) \\
\leq & V\left(0, i_{0}, x(0)-D(i, x(-\tau))\right) \\
& +\int_{0}^{t}\left(\gamma(s)-W_{1}(x(s))+W_{2}(x(s-\tau))\right. \\
\leq & V\left(0, i_{0}, x(0)-D(i, x(-\tau))\right) \\
& +\int_{0}^{t} \gamma(s) d s-\int_{0}^{t} W_{1}(x(s)) d s+\int_{0}^{t} W_{2}(x(s-\tau)) d s \\
& -\int_{0}^{t} W_{3}(x(s)-D(r(s), x(s-\tau))) d s+M(t) \\
= & V\left(0, i_{0}, x(0)-D(i, \xi(-\tau))\right) \\
& +\int_{0}^{t} \gamma(s) d s-\int_{0}^{t}\left(W_{1}(x(s))-W_{2}(x(s))\right) d s \\
& +\int_{0}^{t} \gamma(s) d s-\int_{0}^{t} W_{1}(x(s)) d s+\int_{-\tau}^{t-\tau} W_{2}(x(s)) d s \\
\leq & -\int_{0}^{t} W_{3}(x(s)-D(r(s), x(s-\tau))) d s+M(t)
\end{aligned}
$$

By the convergence theorem of nonnegative semimartingales (Lemma 7), we have (A.1).

Step 2. We prove

$$
\sup _{0 \leq t<\infty}|x(t)|<\infty \text { a.s. }
$$

Indeed, from (A.1), we have

$$
\sup _{0 \leq t<\infty} V(t, r(t), x(t)-D(r(t), x(t-\tau)))<\infty \text { a.s. }
$$

which together with $(\mathrm{C} 1)$ yields

$$
\sup _{0 \leq t<\infty}|x(t)-D(r(t), x(t-\tau))|<\infty \text { a.s. }
$$

Now, for any $T>0$, by $(\mathrm{H} 2)$, we have that if $0 \leq t \leq T$, then

$$
\begin{aligned}
& |x(t)| \\
& \quad \leq|D(r(t), x(t-\tau))|+|x(\mathrm{t})-D(r(t), x(t-\tau))| \\
& \quad \leq \kappa|x(t-\tau)|+|x(t)-D(r(t), x(t-\tau))| .
\end{aligned}
$$


where $\kappa=\max _{i \in S} \kappa_{i}<1$. This implies

$$
\begin{aligned}
& \sup _{0 \leq t \leq T}|x(t)| \\
& \quad \leq \kappa \sup _{0 \leq t \leq T}|x(t-\tau)|+\sup _{0 \leq t \leq T}|x(t)-D(r(t), x(t-\tau))| \\
& \quad=\kappa \beta+\kappa \sup _{0 \leq t \leq T}|x(t)|+\sup _{0 \leq t \leq T}|x(t)-D(r(t), x(t-\tau))| .
\end{aligned}
$$

where $\beta$ is the bound for the initial data $\xi$. Hence

$$
\begin{aligned}
& \sup _{0 \leq t \leq T}|x(t)| \\
& \quad \leq \frac{1}{1-\kappa}\left(\kappa \beta+\sup _{0 \leq t \leq T}|x(t)-D(r(t), x(t-\tau))|\right) .
\end{aligned}
$$

Letting $T \rightarrow \infty$ and using (A.6), we obtain (A.4).

Step 3. We prove

$$
\lim _{t \rightarrow \infty} W_{3}(x(t)-D(r(t), x(t-\tau)))=0 \text { a.s. }
$$

In fact, taking the expectations on both sides of (A.3) and letting $t \rightarrow \infty$, we obtain that

$$
\mathbb{E} \int_{0}^{\infty} W(s) d s<\infty,
$$

where $W(s)=W_{1}(x(s))-W_{2}(x(s))+W_{3}(z(s)), z(s)=x(s)-$ $D(r(s), x(s-\tau))$.

This implies

$$
\int_{0}^{\infty} W(s) d s<\infty \text { a.s. }
$$

or equivalently

$$
\begin{gathered}
\int_{0}^{\infty}\left(W_{1}(x(s))-W_{2}(x(s))\right) d s<\infty \text { a.s. } \\
\int_{0}^{\infty} W_{3}(z(s)) d s<\infty \text { a.s. }
\end{gathered}
$$

From (A.12), we have

$$
\underbrace{\liminf }_{t \rightarrow \infty} W(t)=0 \text { a.s. }
$$

or equivalently

$$
\begin{gathered}
\varliminf_{t \rightarrow \infty}^{\operatorname{iminf}}\left(W_{1}(x(t))-W_{2}(x(t))=0\right. \text { a.s. } \\
\underbrace{\liminf }_{t \rightarrow \infty} W_{3}(z(t))=0 \text { a.s. }
\end{gathered}
$$

Now we will prove (A.10): $\lim _{t \rightarrow \infty} W_{3}(z(t))=0$ a.s. In fact, if (A.10) is false, then

$$
\mathbb{P}\{\underbrace{\limsup }_{t \rightarrow \infty} W_{3}(z(t))>0\}>0 .
$$

Hence there is a number $\varepsilon>0$ such that

$$
\mathbb{P}\left(\Omega_{1}\right) \geq 3 \varepsilon
$$

where $\Omega_{1}=\{\underbrace{\limsup }_{t \rightarrow \infty} W_{3}(z(t))>2 \varepsilon\}$.

Recalling (A.4), as well as the boundedness of the initial data $\xi$, we can find a positive number $h$, which depends on $\varepsilon$, sufficiently large for

$$
\mathbb{P}\left(\Omega_{2}\right) \geq 1-\varepsilon,
$$

where $\Omega_{2}=\left\{\sup _{-\tau \leq t<\infty}|z(t)|<h\right\}$.

It is easy to see from (A.17) and (A.18) that

$$
\mathbb{P}\left(\Omega_{1} \cap \Omega_{2}\right) \geq 2 \varepsilon
$$

We now define a sequence of stopping times as follows:

$$
\begin{gathered}
\tau_{h}=\inf \{t \geq 0:|x(t)| \wedge|z(t)| \geq h\} \\
\sigma_{1}=\inf \left\{t \geq 0: W_{3}(z(t)) \geq 2 \varepsilon\right\} \\
\sigma_{2 k}=\left\{t \geq \sigma_{2 k-1}: W_{3}(z(t))<\varepsilon\right\}, \quad k=1,2, \ldots \\
\sigma_{2 k+1}=\left\{t \geq \sigma_{2 k}: W_{3}(z(t)) \geq 2 \varepsilon\right\}, \quad k=1,2, \ldots,
\end{gathered}
$$

where, throughout this paper, we set inf $\emptyset=\infty$.

From (A.14) and the definition of $\Omega_{1}$ and $\Omega_{2}$, we observe that if $\omega \in \Omega_{1} \cap \Omega_{2}$, then

$$
\tau_{h}=\infty, \quad \sigma_{k}<\infty \quad \forall k \geq 1 .
$$

Let $I_{A}$ denote the indication function of set $A$. Noting the fact that $\sigma_{2 k}<\infty$, whenever $\sigma_{2 k-1}<\infty$, we can derive from (A.11) that

$$
\begin{aligned}
\infty & >\mathbb{E} \int_{0}^{\infty} W_{3}(z(t)) d t \\
& \geq \sum_{k=1}^{\infty} \mathbb{E}\left[I_{\sigma_{2 k-1}<\infty, \sigma_{2 k}<\infty, \tau_{h}=\infty} \int_{\sigma_{2 k-1}}^{\sigma_{2 k}} W_{3}(z(t)) d t\right] \\
& \geq \varepsilon \sum_{k=1}^{\infty} \mathbb{E}\left[I_{\sigma_{2 k-1}<\infty, \tau_{h}=\infty}\left(\sigma_{2 k}-\sigma_{2 k-1}\right)\right] .
\end{aligned}
$$

On the other hand, by (H1), there exists a constant $K_{h}>0$, such that

$$
|f(t, i, x, y)|^{2} \vee|g(t, i, x, y)|^{2} \leq K_{h}^{2}
$$

whenever $|x| \vee|y|<h$ and $(t, i) \in R_{+} \times S$.

By the Hölder inequality (Lemma 8 ) and the Doob martingale inequality (Lemma 9), we compute that, for any $T>0$ and $k=1,2, \ldots$,

$$
\begin{gathered}
\mathbb{E}\left[I_{\tau_{h} \wedge \sigma_{2 k-1}<\infty} \sup _{0 \leq t \leq T} \mid z\left(\tau_{h} \wedge\left(\sigma_{2 k-1}+t\right)\right)\right. \\
\left.\quad-\left.z\left(\tau_{h} \wedge \sigma_{2 k-1}\right)\right|^{2}\right] \leq 2 K_{h}^{2} T(T+4) .
\end{gathered}
$$


Since $W_{3}(\cdot)$ is continuous in $\mathbb{R}^{n}$, there exists a closed ball $\bar{S}_{h}=$ $\left\{x \in \mathbb{R}^{n}:|x|<h\right\}$ such that $W_{3}(\cdot)$ is uniformly continuous in $\bar{S}_{h}$. We can therefore choose $\delta=\delta(\varepsilon)>0$ so small such that

$$
\left|W_{3}(x)-W_{3}(y)\right|<\frac{\varepsilon}{2} \quad \text { whenever } x, y \in \bar{S}_{h},|x-y|<\delta \text {. }
$$

We furthermore choose $T=T(\varepsilon, \delta, h)>0$ sufficiently small for

$$
\frac{2 K_{h}^{2} T(T+4)}{\delta^{2}}<\varepsilon
$$

It then follows from (A.24) and Chebyshev's inequality (Lemma 10) that

$$
\begin{gathered}
\mathbb{P}\left(\{ \sigma _ { 2 k - 1 } \wedge \tau _ { h } < \infty \} \cap \left\{\sup _{0 \leq t \leq T} \mid z\left(\tau_{h} \wedge\left(\sigma_{2 k-1}+t\right)\right)\right.\right. \\
\left.-z\left(\tau_{h} \wedge \sigma_{2 k-1}\right) \mid \geq \delta\right) \leq \frac{1}{\delta^{2}}\left(2 K_{h}^{2} T(T+4)\right)<\varepsilon .
\end{gathered}
$$

Note that

$$
\begin{aligned}
& \left\{\sigma_{2 k-1}<\infty, \tau_{h}=\infty\right\}=\left\{\tau_{h} \wedge \sigma_{2 k-1}<\infty, \tau<\infty\right\} \\
& \quad \subseteq\left\{\tau_{h} \wedge \sigma_{2 k-1}<\infty\right\} .
\end{aligned}
$$

We hence have

$$
\begin{aligned}
& \mathbb{P}\left(\left\{\sigma_{2 k-1}<\infty, \tau_{h}=\infty\right\}\right. \\
& \left.\cap\left\{\sup _{0 \leq t \leq T}\left|z\left(\sigma_{2 k-1}+t\right)-z\left(\sigma_{2 k-1}\right)\right| \geq \delta\right\}\right)<\varepsilon .
\end{aligned}
$$

By (A.19) and (A.21), we further compute

$$
\begin{aligned}
\mathbb{P}\left(\left\{\sigma_{2 k-1}<\infty, \tau_{h}=\infty\right\}\right. \\
\left.\quad \cap\left\{\sup _{0 \leq t \leq T}\left|z\left(\sigma_{2 k-1}+t\right)-z\left(\sigma_{2 k-1}\right)\right|<\delta\right\}\right) \\
=\mathbb{P}\left(\left\{\sigma_{2 k-1}<\infty, \tau_{h}=\infty\right\}\right) \\
\quad \times \mathbb{P}\left(\left\{\sigma_{2 k-1}<\infty, \tau_{h}=\infty\right\}\right. \\
\quad \cap\left\{\sup _{0 \leq t \leq T} \mid z\left(\sigma_{2 k-1}+t\right)\right. \\
\left.\left.-z\left(\sigma_{2 k-1}\right) \mid \geq \delta\right\}\right)>2 \varepsilon-\varepsilon=\varepsilon .
\end{aligned}
$$

By (A.25), we hence obtain that

$$
\begin{gathered}
\mathbb{P}\left(\left\{\sigma_{2 k-1}<\infty, \tau_{h}=\infty\right\}\right. \\
\cap\left\{\sup _{0 \leq t \leq T} \mid W_{3}\left(z\left(\sigma_{2 k-1}+t\right)\right)\right. \\
\left.\left.\quad-W_{3}\left(z\left(\sigma_{2 k-1}\right)\right) \mid<\varepsilon\right\}\right)>\varepsilon .
\end{gathered}
$$

Set

$$
\bar{\Omega}_{k}=\left\{\sup _{0 \leq t \leq T}\left|W_{3}\left(z\left(\sigma_{2 k-1}+t\right)\right)-W_{3}\left(z\left(\sigma_{2 k-1}\right)\right)\right|<\varepsilon\right\} .
$$

Note that

$$
\sigma_{2 k}(\omega)-\sigma_{2 k-1}(\omega) \geq T \quad \text { if } \omega \in\left\{\sigma_{2 k-1}<\infty, \tau_{h}=\infty\right\} \cap \bar{\Omega}_{k} .
$$

We derive from (A.22) and (A.31) that

$$
\begin{aligned}
& \infty>\varepsilon \sum_{k=1}^{\infty} \mathbb{E}\left[I_{\left(\sigma_{2 k-1}<\infty, \tau_{h}=\infty\right)}\left(\sigma_{2 k}-\sigma_{2 k-1}\right)\right] \\
& \geq \varepsilon \sum_{k=1}^{\infty} \mathbb{E}\left[I_{\left(\sigma_{2 k-1}<\infty, \tau_{h}=\infty\right) \cap \bar{\Omega}_{k}}\left(\sigma_{2 k}-\sigma_{2 k-1}\right)\right] \\
& \geq \varepsilon T \sum_{k=1}^{\infty} \mathbb{P}\left(\left\{\sigma_{2 k-1}<\infty, \tau_{h}=\infty\right\} \cap \bar{\Omega}_{k}\right) \\
& \geq \varepsilon T \sum_{k=1}^{\infty} \varepsilon=\infty
\end{aligned}
$$

which is a contradiction. So (A.10) must hold.

Step 4 . We prove that $\operatorname{Ker}\left(W_{3}\right) \neq \emptyset$ and

$$
\begin{aligned}
& \lim _{t \rightarrow \infty} d\left(x\left(t ; \xi, i_{0}\right)-D\left(x\left(t-\tau ; \xi, i_{0}\right), r(t)\right), \operatorname{Ker}\left(W_{3}\right)\right) \\
& \quad=0 \text { a.s. }
\end{aligned}
$$

By (A.10) and (A.6), we see that there is an $\Omega_{0} \subset \Omega$ with $\mathbb{P}\left(\Omega_{0}\right)=1$ such that

$$
\lim _{t \rightarrow \infty} W_{3}(z(t, \omega))=0, \quad \sup _{0 \leq t<\infty}|z(t, \omega)|<\infty \quad \forall \omega \in \Omega_{0} .
$$

Choose any $\omega \in \Omega_{0}$. Then $\{z(t, \omega)\}_{t \geq 0}$ is bounded in $\mathbb{R}^{n}$ so there must be an increasing sequence $\left\{\mathrm{t}_{k}\right\}_{k \geq 1}$ such that $t_{k} \rightarrow$ $\infty$ and $\left\{z\left(t_{k}, \omega\right)\right\}_{k \geq 1}$ converges to some $\bar{z} \in \mathbb{R}^{n}$. Thus

$$
W_{3}(\bar{z})=\lim _{k \rightarrow \infty} W_{3}\left(z\left(t_{k}, \omega\right)\right)=0
$$

which implies that $\bar{z} \in \operatorname{Ker}\left(W_{3}\right)$ whence $\operatorname{Ker}\left(W_{3}\right) \neq \emptyset$. From this, we can show that

$$
\lim _{t \rightarrow \infty} d\left(z(t, \omega), \quad \operatorname{Ker}\left(W_{3}\right)\right)=0 \quad \forall \omega \in \Omega_{0} .
$$


If this is false, then there is some $\bar{\omega} \in \Omega_{0}$ such that

$$
\underbrace{\limsup }_{t \rightarrow \infty} d\left(z(t, \bar{\omega}), \quad \operatorname{Ker}\left(W_{3}\right)\right)>0 .
$$

Hence there is a subsequence $\left\{z\left(t_{k}, \bar{\omega}\right)\right\}_{k \geq 0}$ of $\{z(t, \bar{\omega})\}_{t \geq 0}$ such that

$$
\lim _{k \rightarrow \infty} d\left(z\left(t_{k}, \bar{\omega}\right), \quad \operatorname{Ker}\left(W_{3}\right)\right)>\bar{\varepsilon}
$$

for some $\bar{\varepsilon}>0$. Since $\left\{z\left(t_{k}, \bar{\omega}\right)\right\}_{k \geq 0}$ is bounded, we can find its subsequence $\left\{z\left(\bar{t}_{k}, \bar{\omega}\right)\right\}_{k \geq 0}$ which converges to $\widehat{z} \in \mathbb{R}^{n}$. Clearly, $\widehat{z} \notin \operatorname{Ker}\left(W_{3}\right)$ so $W_{3}(\widehat{z})>0$. But, by (A.36),

$$
W_{3}(\widehat{z})=\lim _{k \rightarrow \infty} W_{3}\left(z\left(\bar{t}_{k}, \bar{\omega}\right)\right)=0
$$

a contradiction. Hence (A.38) must hold and (A.35) holds yet.

Step 5. We prove (R2).

Under the assumption that

$$
W_{3}(x)=0 \quad \text { iff } x=0,
$$

we have $\operatorname{Ker}\left(W_{3}\right)=\{0\}$. It then follows from (A.35) that

$$
\lim _{t \rightarrow 0}[x(t)-D(x(t-\tau), r(t))]=\lim _{t \rightarrow 0} z(t)=0 \text { a.s. }
$$

But, by (H2),

$$
\begin{aligned}
& |x(t)| \\
& \quad \leq|D(x(t-\tau), r(t))|+|x(t)-D(x(t-\tau), r(t))| \\
& \quad \leq \kappa|x(t-\tau)|+|x(t)-D(x(t-\tau), r(t))|,
\end{aligned}
$$

where $\kappa \in(0,1)$ has been defined above. Letting $t \rightarrow \infty$, we obtain that

$$
\underbrace{\limsup }_{t \rightarrow \infty}|x(t)| \leq \kappa \underbrace{\limsup }_{t \rightarrow \infty}|x(t)| \text { a.s. }
$$

This together with (A.4) yields

$$
\lim _{t \rightarrow \infty}|x(t)|=0 \text { a.s. }
$$

which is (32) and the proof is therefore completed.

\section{Conflict of Interests}

The authors declare that there is no conflict of interests regarding the publication of this paper.

\section{Acknowledgments}

This work is partially supported by the National Natural Science Foundation of China under Grant no. 61203337, the Innovation Program of Shanghai Municipal Education Commission (12zz064), the Specialized Research Fund for the Doctoral Program of Higher Education under Grant no. 20120075120009, the Natural Science Foundation of Shanghai under Grant no. 12ZR1440200, the Fundamental Research Funds for the Central Universities (2232012D3-18), and DHU Distinguished Young Professor Program (B201309).

\section{References}

[1] Y. Tang and W. K. Wong, "Distributed synchronization of coupled neural networks via randomly occurring control," IEEE Transactions on Neural Networks and Learning Systems, vol. 24, no. 3, pp. 435-447, 2013.

[2] Z. Wu, P. Shi, H. Su, and J. Chu, "Exponential synchronization of neural networks with discrete and distributed delays under time-varying sampling," IEEE Transactions on Neural Networks and Learning Systems, vol. 23, no. 9, pp. 1368-1376, 2012.

[3] Z. Wu, P. Shi, H. Su, and J. Chu, "Stochastic synchronization of Markovian jump neural networks with time-varying delay using sampled-data," IEEE Transactions on Cybernetics, vol. 23, no. 6, pp. 1796-1806, 2013.

[4] Z. Wu, P. Shi, H. Su, and J. Chu, "Passivity analysis for discretetime stochastic markovian jump neural networks with mixed time delays," IEEE Transactions on Neural Networks, vol. 22, no. 10, pp. 1566-1575, 2011.

[5] J. Lu, W. C. Ho, J. Cao, and J. Kurths, "Exponential synchronization of linearly coupled neural networks with impulsive disturbances," IEEE Transactions on Neural Networks, vol. 22, no. 2, pp. 329-336, 2011.

[6] J. Lu, D. W. C. Ho, J. Cao, and J. Kurths, "Single impulsive controller for globally exponential synchronization of dynamical networks," Nonlinear Analysis. Real World Applications, vol. 14, no. 1, pp. 581-593, 2013.

[7] J. Lu, J. Kurths, J. Cao, N. Mahdavi, and C. Huang, "Synchronization control for nonlinear stochastic dynamical networks: pinning impulsive strategy," IEEE Transactions on Neural Networks and Learning Systems, vol. 23, no. 2, pp. 285-292, 2012.

[8] J. Lu, D. W. C. Ho, J. Cao, and J. Kurths, "Exponential synchronization of linearly coupled neural networks with impulsive disturbances," IEEE Transactions on Neural Networks, vol. 22, no. 2, pp. 329-335, 2011.

[9] Y. Tang, R. Qiu, J.-a. Fang, Q. Miao, and M. Xia, "Adaptive lag synchronization in unknown stochastic chaotic neural networks with discrete and distributed time-varying delays," Physics Letters A, vol. 372, no. 24, pp. 4425-4433, 2008.

[10] Y. Sun and J. Cao, "Adaptive lag synchronization of unknown chaotic delayed neural networks with noise perturbation," Physics Letters A General, Atomic and Solid State Physics, vol. 364, no. 3-4, pp. 277-285, 2007.

[11] X. Li and J. Cao, "Adaptive synchronization for delayed neural networks with stochastic perturbation," Journal of the Franklin Institute. Engineering and Applied Mathematics, vol. 345, no. 7, pp. 779-791, 2008.

[12] W. Zhou, D. Tong, Y. Gao, C. Ji, and H. Su, "Mode and delay-dependent adaptive exponential synchronization in pth moment for stochastic delayed neural networks with Markovian switching," IEEE Transactions on Neural Networks and Learning System, vol. 23, no. 4, pp. 662-668, 2012.

[13] X. Ding, Y. Gao, W. Zhou, D. Tong, and H. Su, "Adaptive almost surely asymptotically synchronization for stochastic delayed neural networks with Markovian switching," Advances in Difference Equations, vol. 195, p. 211, 2013.

[14] X. Li, "Global robust stability for stochastic interval neural networks with continuously distributed delays of neutral type," Applied Mathematics and Computation, vol. 215, no. 12, pp. 4370-4384, 2010.

[15] J. H. Park, "Synchronization of cellular neural networks of neutral type via dynamic feedback controller," Chaos, Solitons and Fractals, vol. 42, no. 3, pp. 1299-1304, 2009. 
[16] X. Mao, Y. Shen, and C. Yuan, "Almost surely asymptotic stability of neutral stochastic differential delay equations with Markovian switching," Stochastic Processes and their Applications, vol. 118, no. 8, pp. 1385-1406, 2008.

[17] V. Kolmanovskii, N. Koroleva, T. Maizenberg, X. Mao, and A. Matasov, "Neutral stochastic differential delay equations with Markovian switching," Stochastic Analysis and Applications, vol. 21, no. 4, pp. 819-847, 2003.

[18] W. Zhou, Y. Gao, D. Tong, C. Ji, and J. Fang, "Adaptive exponential synchronization in pth moment of neutral-type neural networks with time delays and Markovian switching," International Journal of Control, Automation, and Systems, vol. 11, no. 4, pp. 845-851, 2013.

[19] Q. Zhu, W. Zhou, D. Tong, and J. Fang, "Adaptive synchronization for stochastic neural networks of neutral-type with mixed time-delays," Neurocomputing, vol. 99, pp. 477-485, 2013.

[20] V. P. Rubanik, Oscillations of Qasilinear Systems with Retardation, Nauka, Moscow, Russia, 1969.

[21] X. Mao and C. Yuan, Stochastic Differential Equations with Markovian Switching, Imperial College Press, London, UK, 2006.

[22] R. Sh. Liptser and A. N. Shiryayev, Theory of Martingales, Kluwer Academic, Dordrecht, The Netherlands, 1989.

[23] C. Yuan and X. Mao, "Robust stability and controllability of stochastic differential delay equations with Markovian switching," Automatica. A Journal of IFAC, the International Federation of Automatic Control, vol. 40, no. 3, pp. 343-354, 2004. 


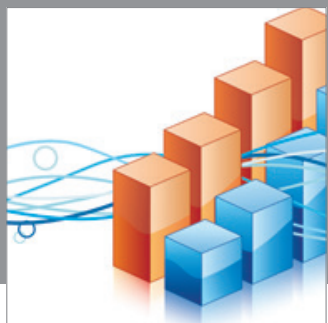

Advances in

Operations Research

mansans

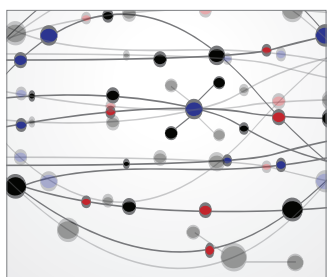

The Scientific World Journal
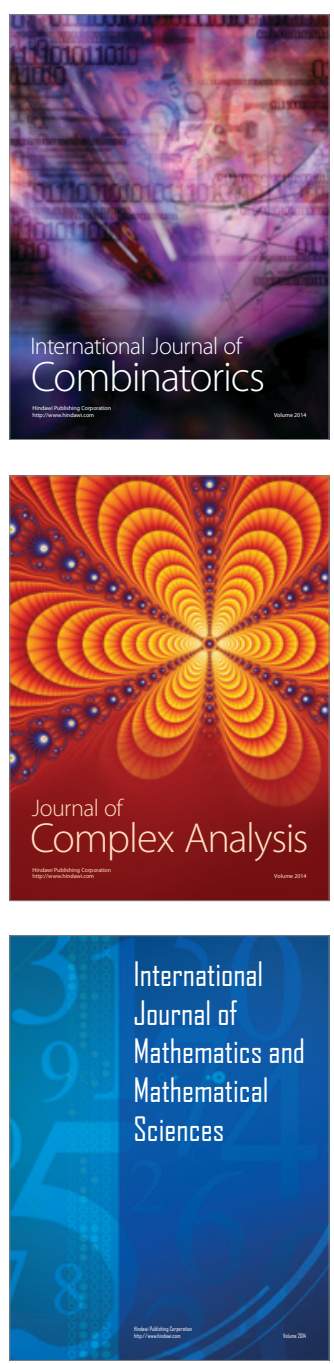
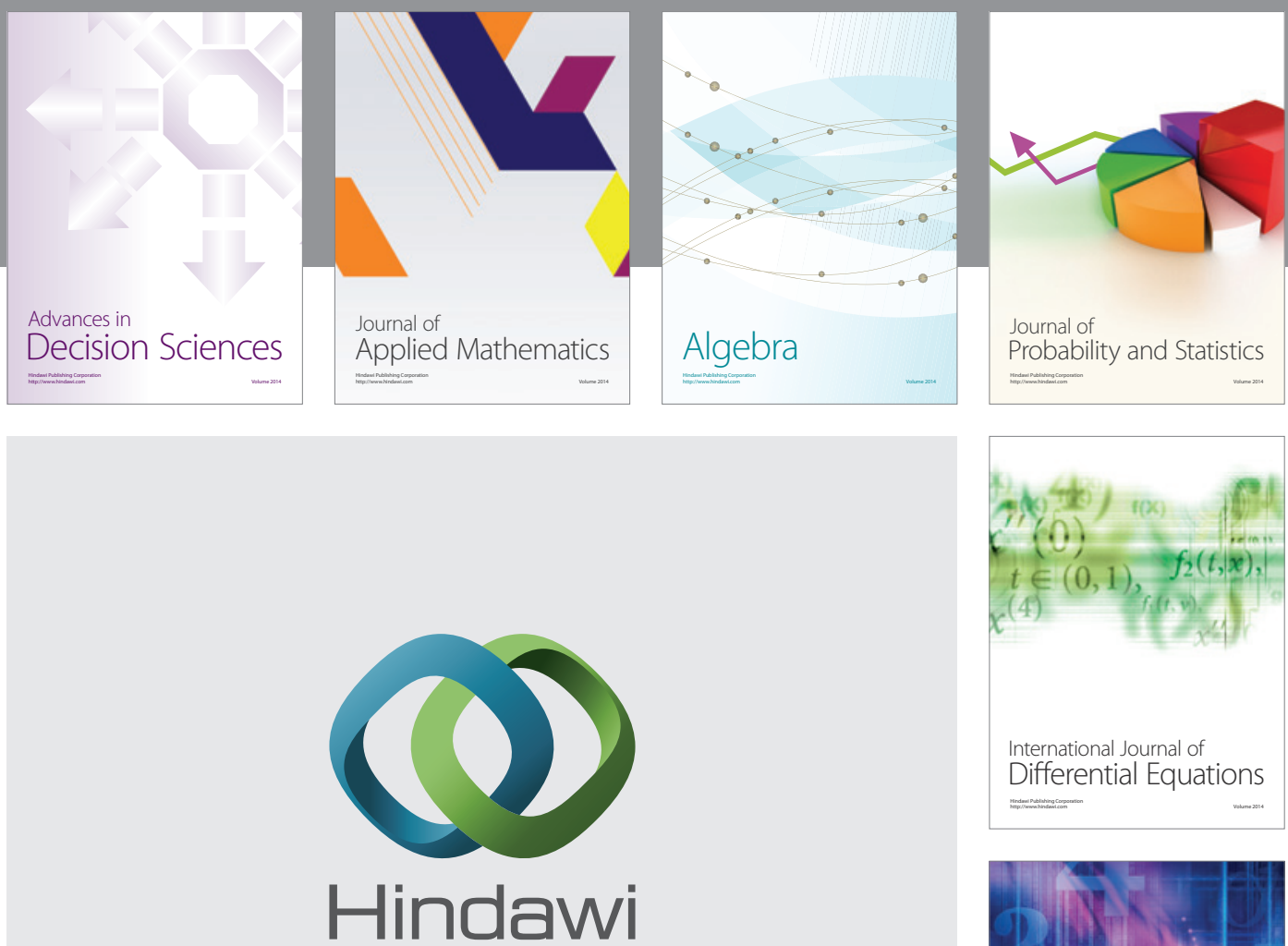

Submit your manuscripts at http://www.hindawi.com
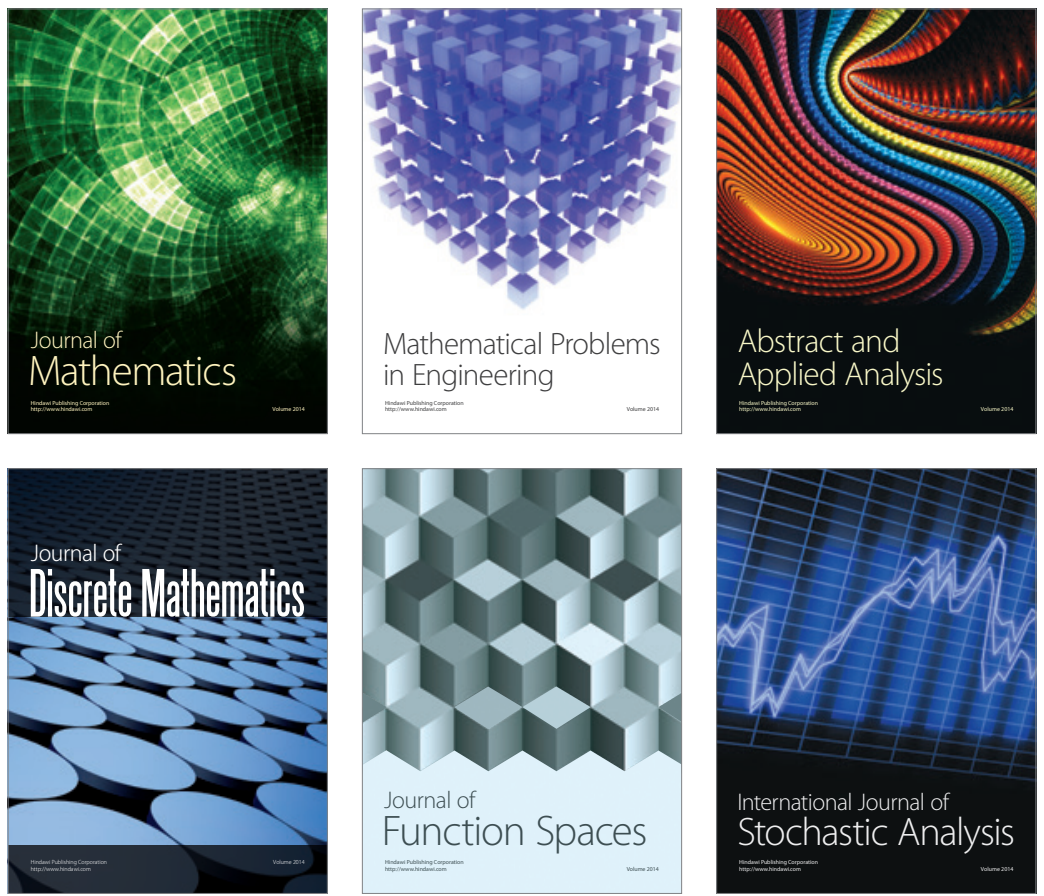

Journal of

Function Spaces

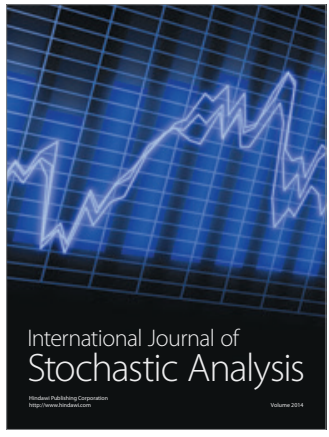

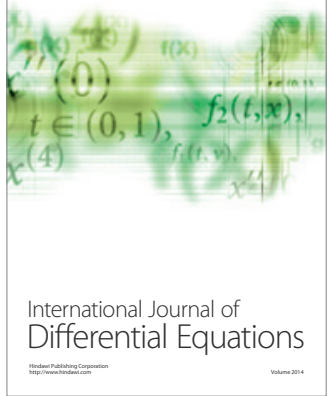
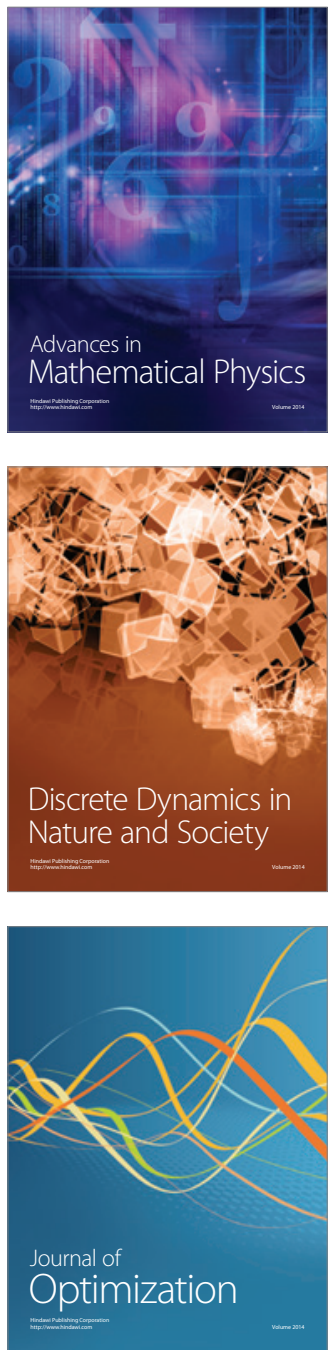